\title{
Tribology in Industry
}

\author{
www.tribology.rs
}

\section{Maximum von Mises Stress in the Sliding Contact of Coated Bodies}

\author{
S. Spinu ${ }^{a}$ \\ a Department of Mechanics and Technologies, Stefan cel Mare University of Suceava, 13th University Street, 720229, \\ Suceava, Romania.
}

Keywords:

Sliding contact

Numerical simulation

Coatings

Fast Fourier transfrom

Convolution

Von Mises equivalent stress

Corresponding author:

\section{S. Spinu}

Department of Mechanics and

Technologies, Stefan cel Mare

University of Suceava, 13th

University Street, 720229, Suceava,

Romania.

E-mail: sergiu.spinu@fim.usv.ro

\begin{abstract}
A B S T R A C T
Competent design decisions for the optimum configuration of a coated contact require the prediction of the stress state developing in the coated body under contact load. Conventional methods of superposition of effects applied in the elasticity of homogenous bodies fail in case of multilayered materials as the response of the latters to point load has only been derived in the frequency domain. A technique for assessment of the elastic response of coated bodies to arbitrary loadings is advanced in this paper, by employing the convolution theorem in the calculation of the stresses and displacements resulting in a sliding contact process. The solution of the elastic frictional contact in full sliding is achieved iteratively, using a state-of-the-art method originally developed for the contact of rough homogenous bodies. Results presented in the literature for the sliding contact of coated bodied are replicated using the newly proposed algorithm. A parametric study is subsequently performed to assess the combined influence of the frictional coefficient, the coating thickness and the elastic modulus mismatch between the coating and the substrate, on the locations and intensity of the maximum von Mises equivalent stress.
\end{abstract}

(C) 2019 Published by Faculty of Engineering

\section{INTRODUCTION}

In a mechanical contact process, the tribological performances of the contacting elements can be enhanced by engaging coating technologies, providing low friction, and high wear and corrosion resistance. Many industrial applications currently involve coatings to extend the service life of engineering elements undergoing contact load. The study of the stresses developing during the contact process is an essential prerequisite to the competent coating design. Analytical solutions for the elastic response of a coated half-space have only been developed in the frequency domain, and are known as the frequency response functions (FRF). Their counterparts in the time/space domain (i.e., the Green's functions) are difficult, if not impossible, to obtain. With the advancement of the discrete Fourier transform applications in the study of the mechanical contact, the use of numerical techniques for coated contacts is tantalizing and promises increased algorithmic efficiency. 
The spectral analysis of contact processes was initiated by these authors [1], providing, by means of the fast Fourier transform (FFT), superior computational efficiency compared to the continuous integral transform method. O'Süllivian and King [2] obtained the FRFs for the stress state in the contact of bi-layered bodies in sliding. They determined distributions of the von Mises stress in the coating, as well as in the substrate. The numerical results suggest that the location of the maximum equivalent stress is influenced by the elastic modulus mismatch between the coating and the substrate, by the value of the frictional coefficient, and also by the relation between the coating thickness and the radius of the contact area. The coated contact of rough surfaces was subsequently studied by Nogi and Kato [3] using the FFT technique. The conjugate gradient method (CGM) was employed to obtain the solution of the contact problem for homogenous or layered bodies. Polonsky and Keer debated [4] the periodicity error related to the application of the FFT to contact problems and subsequently developed [5] a strategy for the analysis of the stress state in layered elastic solids containing cracks. The cause of the periodicity error was further studied by Liu et al. [6], resulting in the DCFFT method that fully circumvents the periodicity error. Liu and Wang [7] applied the latter method to derive the elastic stresses induced by the contact surface tractions, whereas Wang et al. [8] investigated, using a similar approach, the partial slip contact of coated bodies. Zhang et al. [9] derived the mapped data allowing the prediction of the positions of plastic yielding initiation in hard coatings. Yu et al. [10] calculated the maximum equivalent stress in trilayer bodies undergoing contact load. More recently, Yu et al. [11] calculated the analytical response of multilayered materials in the Fourier domain, and studied the contact process of multilayered bodies having varied coating arrangements.

The knowledge of the intensity and position of the maximum von Mises equivalent stress is critical information for the propensity of plastic yield initiation or crack nucleation in a coated contact, thus guiding the optimal design of coatings without contact failure. Considering the mathematical complexity of the arising contact model and the lack of analytical solutions, the goal of this work is to investigate the combined influence of the coating thickness, frictional coefficients and elastic modulus mismatch on the maximum von Mises stress and its location, thus providing a map of the possible yield locations from a novel parametric study performed for a wide range of bilayer materials.

\section{CONTACT MODEL}

The schematic of the contact model is depicted in Fig. 1. A Cartezian coordinate system with the $x$ and $y$ axes laying in the plane that separates best the bounding surfaces of the contacting solids is employed. As the contact is established and a normal force is transmitted, the bodies deform elastically, surface particles within each body are compressed along the normal direction, so that the initial point of contact evolves into a contact region accommodating a mutual contact pressure denoted by $p(x, y)$. The static force equilibrium in the normal direction yields:

$$
W=\int_{-\infty}^{\infty} \int_{-\infty}^{\infty} p(x, y) d x d y
$$

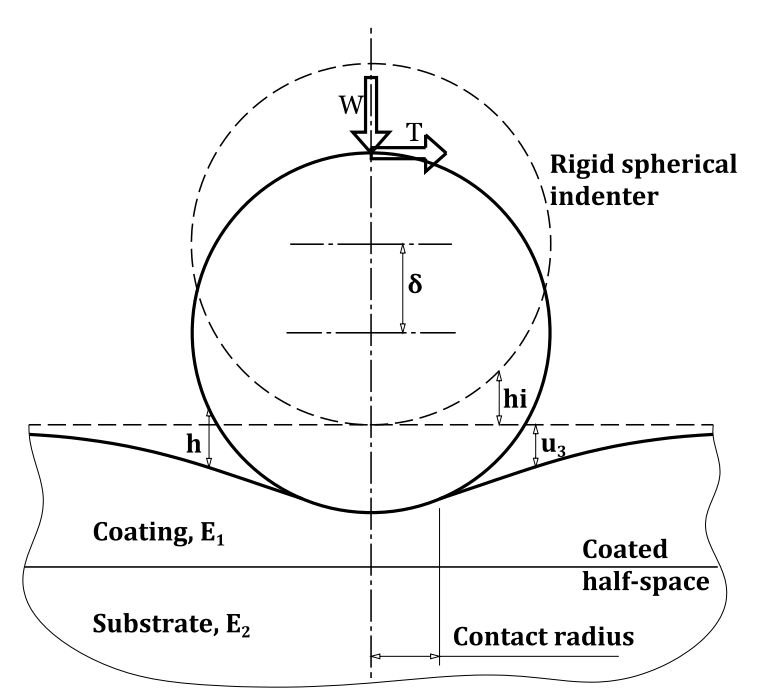

Fig. 1. Representation of a sliding spherical contact progression.

The calculation of the separation $h(x, y)$ between the deformed bodies results by equating the parameters of the contact geometry before and after the deformation:

$$
h(x, y)+\delta=u_{3}(x, y)+h i(x, y),
$$

where $h i$ denotes the gap in unloaded state (depicted using dashed lines in Fig. 1), $u_{3}$ the 
composite (i.e., relative) displacement induced by the pressure $p$ in the direction of the $x_{3}$-axis, and $\delta$ the rigid-body approach, i.e. the approach of points within the elastic bodies distant to the contact region. Equations (1) and (2), governing the dry frictionless contact under normal load, hold true for the sliding contact as well, provided the normal displacement $u_{3}$ is calculated considering the contribution of both normal and shear tractions.

The resolution of the normal contact model (1)(2) is difficult to achieve (with the Hertz contact as one notable exception) because neither the pressure distribution, nor the contact area, are known in advance. A numerical solution is expected to improve the prediction capacity for the contact process and deliver support for competent design decisions in important engineering applications. The numerical treatment of the contact model involves a trial-and-error approach, in which a guess computational domain $\Gamma$ is discretised using cuboidal elements having the same magnitude. Problem parameters, e.g. stresses and displacements, are computed only at the centre of each element, and the resulting discrete values are considered instead of the continuous distributions. This discretization strategy allows for computation of displacement and stresses based on the fundamental solutions (i.e., the Green's functions) calculated for unit forces acting in an elastic half-space. Moreover, the integrals in the static force equilibrium equations or in the displacement and stress relations are substituted by summation. To achieve a computational form, the model of the normal contact problem is completed with the boundary conditions (3)-(4), expressing the non-negativity of pressure and the impenetrability of the contacting bodies [12]. In the discrete model, problem parameters can be denoted by using discrete indexes instead of continuous coordinates:

$$
\begin{gathered}
h(k, \ell)=0, \quad p(k, \ell)>0,(k, \ell) \in A ; \\
h(k, \ell)>0, \quad p(k, \ell)=0,(k, \ell) \in \Gamma-A ; \\
h(k, \ell)+\delta=u_{3}(k, \ell)+h i(k, \ell),(k, \ell) \in \Gamma ; \\
W=\sum_{(k, \ell) \in \Gamma} p(k, \ell),
\end{gathered}
$$

where $A$ denotes a discrete substitute of the contact area, consisting in the set of surface elementary patches with positive pressure:

$$
A=\{(k, \ell) \in \Gamma \mid p(k, \ell)>0\} .
$$

The latter assumption assures model reduction to the minimization of a quadratic form whose convergence is guaranteed. The neglect of adhesion (i.e., negative normal contact tractions are not allowed on the contact area) can be considered reasonable in case of metallic bodies, whose real contact area is aggregated from discrete contacts between the asperity peaks. The existence and uniqueness of the solution for the discrete contact model (3)-(6) was discussed in [13].

Provided a method for the calculation of displacement in layered materials, induced by arbitrary, yet known, contact stresses, is made available, the solution of the coated contact model can be achieved in the same manner as for homogenous bodies, as described in [12]. Equation (5) gives rise to a linear system of equations having the nodal pressures as unknowns. A comparative review [15] of the various numerical methods employed in the literature for the resolution of the latter system indicates the conjugate gradient based solver proposed by Polonsky and Keer [12] as the fastest solution method for large systems. The key to the computational efficiency of this algorithm consists in the manner the boundary conditions (3) and (4), as well as the astatic force equilibrium (6), are enforced during the conjugate gradient iterations. A detailed algorithm description is beyond the point of this study, therefore only the algorithm flow-chart is presented in Fig. 2.

The sliding contact model, normally involving the resolution of the contact problem in the tangential direction as well, may be simplified considerably by overlooking the normal displacement due to shear. The latter displacement is usually small compared to its counterpart related to the contact pressure. In this manner, the solution of the sliding contact process can be divided into two steps: (a) the resolution of the frictionless normal contact problem, and (b) the subsequent computation of the frictional shear stresses, which are connected to pressure by the Coulomb law. 


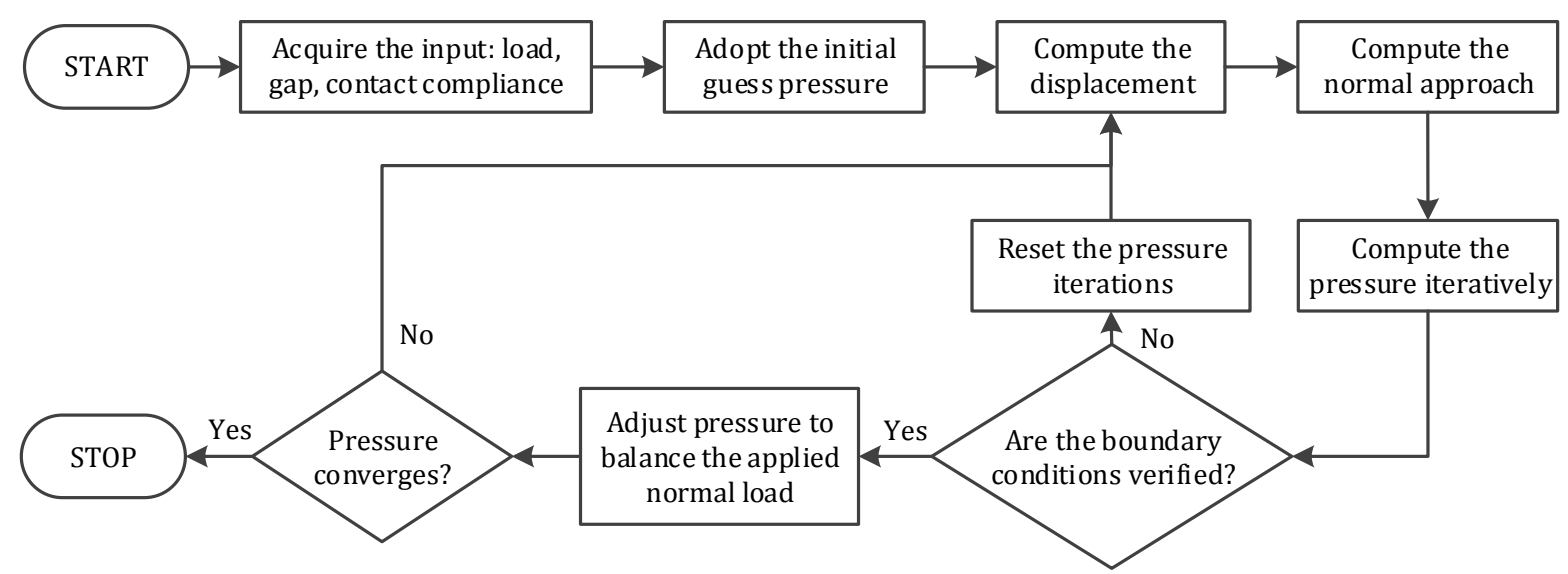

Fig. 2. Algorithm flowchart for solving the frictionless normal contact of layered or homogenous elastic bodies.

Within this framework, the contact process in the normal direction is decoupled from the tangential effects, and the contact pressure is obtained in one step, by solving a frictionless contact scenario. Without this assumption, the numerical solution can still be achieved, but with the computational cost of an additional level of iterations, aiming to stabilise the contact pressure with respect to the normal displacement induced by the shear tractions. The literature $[2,7,9]$ of the sliding contact often employs the aforementioned simplification, as a reasonable compromise between precision and computational efficiency. Consequently, in this study, the shear contact tractions, computed by the kinetic friction formula, are assumed not to affect the pressure distribution. The subsurface stress state results by superimposing stresses due to the shear tractions to those computed in a frictionless contact process. Provided a method for calculation of displacement and stresses in layered materials is available, the numerical methodology for the treatment of the contact state of homogenous materials applies in full to coated bodies. Such a method is advanced in the following section.

\section{DISPLACEMENT AND STRESSES IN COATED BODIES}

The Green's function method can be applied in the framework of the Linear Theory of Elasticity to derive the response of a half-space to general loadings. Stresses and displacements are calculated as integrals resulting from superposition of effects of unit point forces acting in the half-space. The resulting integrals are in fact convolutions products between the half-space response (i.e., the Green's functions), and the excitation (i.e., pressure or shear stress). Whereas a Green's function defines the halfspace response in the space domain, its transform in the Fourier domain is referred to as the frequency response function (FRF). In case of homogenous materials both the Green's functions and the FRFs are expressed in closedform. To our best knowledge, the response of coated bodies has only been derived in the frequency domain as the FRFs. Moreover, the associated Green's functions are difficult, if not impossible, to express.

However, the existence of the analytical solutions for the FRFs of coated bodies encourages the computation of the coated halfspace response directly in the frequency domain, via the discrete convolution theorem [6]. Such methodology is also convenient from the point of view of algorithmic efficiency: the computational complexity of the convolution calculation decreases, for series with $N$ members, from $O\left(N^{2}\right)$ in the space domain, to $O(N \log N)$ in the frequency domain. The discrete convolution theorem, stating that a convolution in the time/space domain can be computed by element-wise multiplication of the Fourier transforms of the convolution terms, is the reason behind this computational advantage.

However, a direct implementation of this methodology induces errors, as the discrete Fourier transform of a discrete series, computed via the fast Fourier transform (FFT), implies periodization with a period equal to the discretized window of observation. From a physical point of view, this periodization implies that not the actual excitation (i.e., the pressure 
or shear series) is accounted for in displacement or stress computation, but a periodical surface load. The spurious neighboring pressure periods may disturb the half-space response, especially at the border of the computational domain. Fortunately, there exist a way of diminishing the effect of this perturbation. Nogi and Kato [3] advanced a robust algorithm for the contact of homogenous or bilayered bodies, with consideration of microtopography, in which the displacement solution was protected against false periodicity by doubling the number of grids along each direction. Liu et al. [6] studied the cause of the periodicity error in the convolution theorem and advanced the DCFFT method that can efficiently handle the transformation of a linear convolution into a discrete cyclic one. A more in-depth study of the error sources, such as the aliasing and the Gibbs phenomena, can be found in [7].

In this paper, the periodicity error is handled by imposing an extension of the target computational domain in each tangential direction, in which the pressure is zero-padded. As suggested in [7], the aliasing phenomena can be reduced by decreasing the sampling interval in the frequency domain. This refinement can be achieved with extended spatial domains having the same spatial sampling interval. A general algorithm for computation of the displacement or stress response of an elastic half-space with known FRFs, is detailed below. Without losing generality, the equations are presented in one dimension only (i.e., the line contact of infinitely long cylinders) for clarity.

The algorithm input consists in: (a) the extent $L$ of the target spatial domain, (b) the excitation series $p_{i}, i=1 \cdots N$, (c) the FRF $\tilde{f}$ of the needed response, and (d) the domain extension ratio $\chi$, chosen with respect to the needed precision and the available computational resources. The adopted notation employs the tilde $(\sim)$ symbol for continuous functions, and the hat $\left({ }^{\wedge}\right)$ for discrete series, both in the frequency domain. Values between 2 and 8 make good candidates for $\chi$. The extent of the resulting extended domain becomes $\chi L$, which is meshed into $\chi N$ elements to preserve the original sampling interval $\Delta=L / N$. The spatial domain extension leads to a refinement of the sampling interval in the frequency domain from $2 \pi / L$ to $2 \pi /(\chi L)$.
The FRF $\tilde{f}$ is evaluated at a series if discrete frequencies $\zeta_{i}$, thus providing a discrete substitute for one member of the convolution product:

$$
\begin{gathered}
\zeta_{i}=2 \pi(i-\chi N / 2) /(\chi L), \quad i=1 \cdots \chi N ; \\
\hat{f}_{i}=\tilde{f}\left(\zeta_{i}\right), \quad i=1 \cdots \chi N .
\end{gathered}
$$

If the evaluation fails due to singularities $\zeta_{\text {sng }}$, the numerically obtained mean value of the FRF can be considered instead. The discrete series $\hat{f}_{i}$ is subsequently rearranged in wrap-around order, and the excitation $p_{i}$ is transferred to the spectral domain after zero-padding, as requested [6] by the discrete cyclic convolution theorem. The spectral response $\hat{\sigma}_{i}$ is obtained as the elementwise product, and the result is transferred to the space domain by means of the inverse fast Fourier transform (IFFT):

$$
\begin{gathered}
\hat{\sigma}_{i}=\hat{f}_{i} \cdot \hat{p}_{i}, \quad i=1 \cdots \chi N ; \\
\sigma=\operatorname{IFFT}(\hat{\sigma}) .
\end{gathered}
$$

In the latter series, having $\chi N$ members, the precision is guaranteed only for the $N$ terms corresponding to the initial target domain.

For a three-dimensional contact problem, the algorithmic computational complexity is dictated by the grid density in the tangential directions, i.e. $N_{1} \times N_{2}$, as well as by the number of layers $N_{3}$ in which the subsurface stresses are evaluated. The convolution products expressing the superposition of effects in the two tangential directions are the most computationally intensive operations. The computational impact is dramatic because these convolutions must be computed two times per iteration in the conjugate gradient method, and twelve times per layer in the subsurface stress computation. The calculation of the convolution integrals in the frequency domain as elementwise products results in a decrease in the computational complexity from $O\left(N_{1}^{2} N_{2}^{2}\right)$ to $O\left(N_{1} N_{2} \log \left(N_{1} N_{2}\right)\right)$. This reduction becomes critical in case of meshes with $N_{1} \times N_{2}>10^{6}$ grids, which is considered as a minimal density for the treatment of rough contact topography. Practically, a contact simulation with $N_{3}=64$, $N_{1}=N_{2}=128$, and $\chi=4$, is finalized on a quadcore $3.2 \mathrm{GHz}$ CPU in less than 30 minutes. 
The aforementioned algorithm can be used to compute the displacement or stress response of a coated half-space to prescribed, but otherwise arbitrary, discrete loading. Integration with the contact model presented in the previous section provides a fully functioning numerical method for the calculation of contact stresses in the sliding contact of coated bodies.

\section{THE FRFS OF THE SHEAR STRESSES}

The mathematical modelling of layered materials was initiated by Burmister [16]. The literature expresses the stress and displacement fields arising in layered materials as functions of the Papkovich-Neuber potentials. The double Fourier transform of these potentials, together with the boundary conditions and the continuity conditions at the layers interface, allows for calculation of the FRFs in the Fourier domain.

In this manner, O'Sullivan and King [2] obtained the stress fields arising in a coated half-space due to normal and shear tractions. The explicit forms of the FRFs in bilayered materials were derived by Nogi and Kato [3] for frictionless normal loading, and by Liu and Wang [7] for both normal and shear loads. Alternative formulas can be found in [8]. Yu et al. [10] derived the FRFs for trilayer materials in contact, whereas $\mathrm{Yu}$ et al. [11] obtained the general form of the FRFs for multilayered materials in a recurrence format.

The FRFs implemented in the numerical program to compute the shear-induced stresses, originally obtained by Liu and Wang [7], are given in equations (12)-(17) for clarity and completeness. In the space domain, stresses in the coated system are expressed in the Cartezian coordinates $x, y$ and $z_{i}, i=1,2$. In the expressions of the FRFs, the index $i=1$ denotes the coating, and $i=2$ the substrate. The shear moduli and the Poisson's ratios for the layer and the substrate are denoted by $G_{i}$ and $v_{i}, i=1,2$. The FRFs $\tilde{\sigma}_{k \ell}$ of the six stress tensor components $\sigma_{k \ell}, k, \ell=x, y, z$, are functions of $\zeta, \xi$ and $z_{1}$ in the coating, and of $\zeta, \xi$ and $z_{2}$ in the substrate, with $\zeta$ and $\xi$ the frequency domain counterparts of the $x$ and $y$ coordinates:

$$
\begin{aligned}
& \tilde{\sigma}_{x x}=-\zeta^{2}\left(D^{(i)} \bar{\varepsilon}^{(i)}+\bar{D}^{(i)} \varepsilon^{(i)}\right)-2 \sqrt{-1} \zeta\left(2-v_{i}\right) \times \\
& \left(B^{(i)} \bar{\varepsilon}^{(i)}+\bar{B}^{(i)} \varepsilon^{(i)}\right)+2 \alpha v_{i}\left(C^{(i)} \bar{\varepsilon}^{(i)}-\bar{C}^{(i)} \varepsilon^{(i)}\right)+ \\
& \sqrt{-1} \zeta^{3} z_{i} \alpha^{-1}\left(B^{(i)} \bar{\varepsilon}^{(i)}-\bar{B}^{(i)} \varepsilon^{(i)}\right)- \\
& z_{i} \zeta^{2}\left(C^{(i)} \bar{\varepsilon}^{(i)}+\bar{C}^{(i)} \varepsilon^{(i)}\right) \text {; } \\
& \tilde{\sigma}_{y y}=-\xi^{2}\left(D^{(i)} \bar{\varepsilon}^{(i)}+\bar{D}^{(i)} \varepsilon^{(i)}\right)-2 \sqrt{-1} \zeta v_{i} \times \\
& \left(B^{(i)} \bar{\varepsilon}^{(i)}+\bar{B}^{(i)} \varepsilon^{(i)}\right)+2 \alpha v_{i}\left(C^{(i)} \bar{\varepsilon}^{(i)}-\bar{C}^{(i)} \varepsilon^{(i)}\right)+ \\
& \sqrt{-1} \zeta \xi^{2} z_{i} \alpha^{-1}\left(B^{(i)} \bar{\varepsilon}^{(i)}-\bar{B}^{(i)} \varepsilon^{(i)}\right)-z_{i} \xi^{2} \times \\
& \left(C^{(i)} \bar{\varepsilon}^{(i)}+\bar{C}^{(i)} \varepsilon^{(i)}\right) ; \\
& \tilde{\sigma}_{z z}=\alpha^{2}\left(D^{(i)} \bar{\varepsilon}^{(i)}+\bar{D}^{(i)} \varepsilon^{(i)}\right)+2 \sqrt{-1} \zeta\left(1-v_{i}\right) \times \\
& \left(B^{(i)} \bar{\varepsilon}^{(i)}+\bar{B}^{(i)} \varepsilon^{(i)}\right)+2 \alpha\left(1-v_{i}\right)\left(C^{(i)} \bar{\varepsilon}^{(i)}-\right. \\
& \left.\bar{C}^{(i)} \varepsilon^{(i)}\right)-\sqrt{-1} \zeta z_{i} \alpha\left(B^{(i)} \bar{\varepsilon}^{(i)}-\bar{B}^{(i)} \varepsilon^{(i)}\right)+ \\
& z_{i} \alpha^{2}\left(C^{(i)} \bar{\varepsilon}^{(i)}+\bar{C}^{(i)} \varepsilon^{(i)}\right) \text {; } \\
& \tilde{\sigma}_{x y}=-\zeta \xi\left(D^{(i)} \bar{\varepsilon}^{(i)}+\bar{D}^{(i)} \varepsilon^{(i)}\right)-2 \sqrt{-1} \xi\left(1-v_{i}\right) \times \\
& \left(B^{(i)} \bar{\varepsilon}^{(i)}+\bar{B}^{(i)} \varepsilon^{(i)}\right)+\sqrt{-1} \zeta^{2} \xi_{z_{i}} \alpha^{-1}\left(B^{(i)} \bar{\varepsilon}^{(i)}-\right. \\
& \left.\bar{B}^{(i)} \varepsilon^{(i)}\right)-z_{i} \zeta \xi\left(C^{(i)} \bar{\varepsilon}^{(i)}+\bar{C}^{(i)} \varepsilon^{(i)}\right) ; \\
& \tilde{\sigma}_{y z}=-\sqrt{-1} \xi \alpha\left(D^{(i)} \bar{\varepsilon}^{(i)}-\bar{D}^{(i)} \varepsilon^{(i)}\right)+\zeta \xi \alpha^{-1} \times \\
& \left(B^{(i)} \bar{\varepsilon}^{(i)}-\bar{B}^{(i)} \varepsilon^{(i)}\right)-\sqrt{-1} \xi\left(1-2 v_{i}\right)\left(C^{(i)} \bar{\varepsilon}^{(i)}+\right. \\
& \left.\bar{C}^{(i)} \varepsilon^{(i)}\right)-\zeta \xi z_{i}\left(B^{(i)} \bar{\varepsilon}^{(i)}+\bar{B}^{(i)} \varepsilon^{(i)}\right)-\sqrt{-1} \xi z_{i} \alpha \times \\
& \left(C^{(i)} \bar{\varepsilon}^{(i)}-\bar{C}^{(i)} \varepsilon^{(i)}\right) \text {; } \\
& \tilde{\sigma}_{z x}=-\sqrt{-1} \zeta \alpha\left(D^{(i)} \bar{\varepsilon}^{(i)}-\bar{D}^{(i)} \varepsilon^{(i)}\right)+\left[\zeta^{2} \alpha^{-1}+\right. \\
& \left.2 \alpha\left(1-v_{i}\right)\right]\left(B^{(i)} \bar{\varepsilon}^{(i)}-\bar{B}^{(i)} \varepsilon^{(i)}\right)-\sqrt{-1} \zeta\left(1-2 v_{i}\right) \times \\
& \left(C^{(i)} \bar{\varepsilon}^{(i)}+\bar{C}^{(i)} \varepsilon^{(i)}\right)-\zeta^{2} z_{i}\left(B^{(i)} \bar{\varepsilon}^{(i)}+\bar{B}^{(i)} \varepsilon^{(i)}\right)- \\
& \sqrt{-1} z_{i} \alpha \zeta\left(C^{(i)} \bar{\varepsilon}^{(i)}-\bar{C}^{(i)} \varepsilon^{(i)}\right) .
\end{aligned}
$$

The above FRFs were defined with the aid of the variables defined in Appendix.

\section{RESULTS AND DISCUSSIONS}

The computer program was first benchmarked against the stress state [2] derived by numerical inverse transformation of the Papkovich-Neuber elastic potentials from the Fourier domain. A sliding spherical contact is considered. The resolution of the normal contact problem provides the hertzian pressure $p_{H}$ and the hertzian contact radius $a_{H}$, which both match well the Hertz theory. The maximum pressure is used as normalizer for stresses, and the contact 
radius for lengths and coordinates. The magnitude of the normal load, the initial gap, the Young modulus of the substrate, $E_{2}=210 \mathrm{GPa}$, and the Poisson's ratios of the elastic materials, $v_{1}=v_{2}=0.3$, are kept constant in all simulations, whereas the coating thickness $h$, the $E_{1} / E_{2}$ ratio and the frictional coefficient $\mu$ are varied.

The pressure distributions profiles predicted for the indentation of a coated half-space by a rigid sphere are presented in Fig. 3. The Hertzian case, plotted for reference, matches well the data corresponding to $E_{1} / E_{2}=1$. The predicted effect of varying the Young's modulus of the layer relative to the substrate compares favourably to that obtained by O'Sülivian and King [2] using a different method, thus validating the computer program.

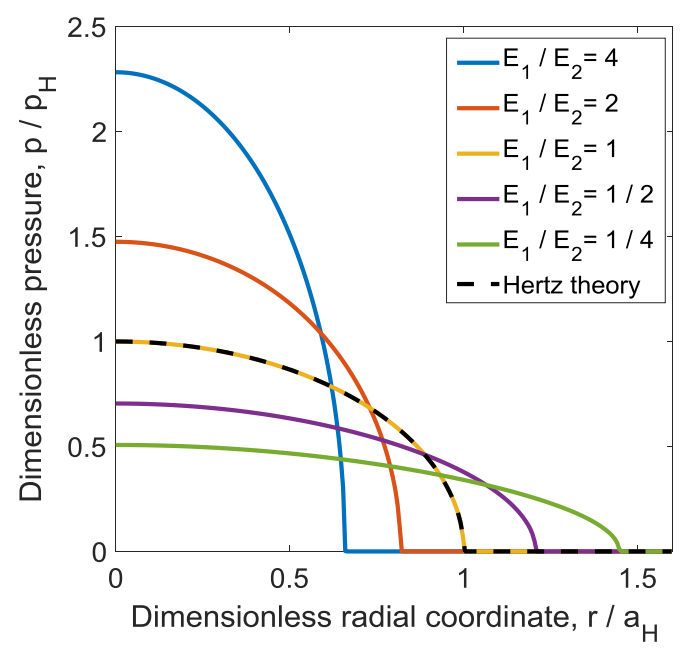

Fig. 3. Indentation of a coated half-space with $h=a_{H}$ by a rigid sphere.

The contour plots of the normalized stress tensor second invariant $\sqrt{J_{2}} / p_{H}$ in the plane $y=0$ are depicted in Figs. 4-6, the location of the maximum value is shown with an $\mathrm{X}$ mark and its magnitude is indicated on each plot. These plots, reproducing closely the ones obtained by O'Süllivian and King [2] through numerical inverse transformation of the Papkovich-Neuber potentials, provide additional program validation. The case $E_{1} / E_{2}=1$ and $\mu=0$ in Fig. 4(a) matches well the Hertz theory.

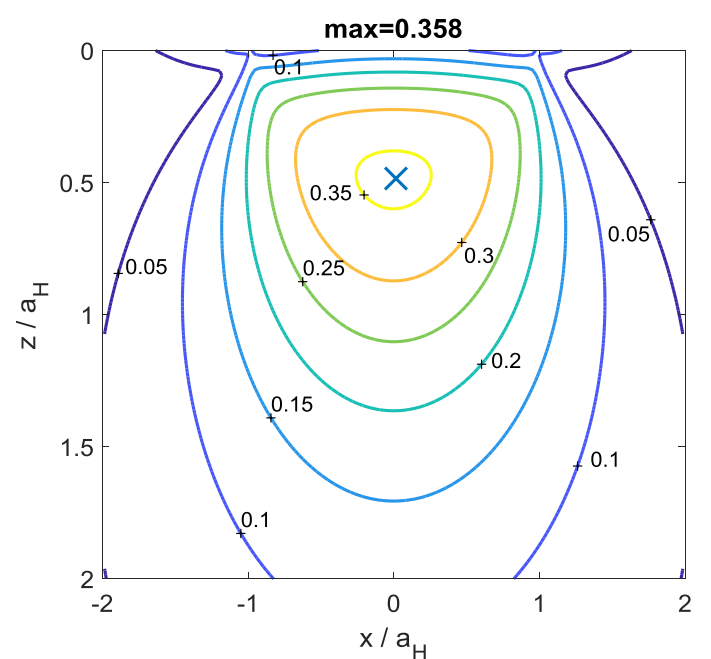

(a)

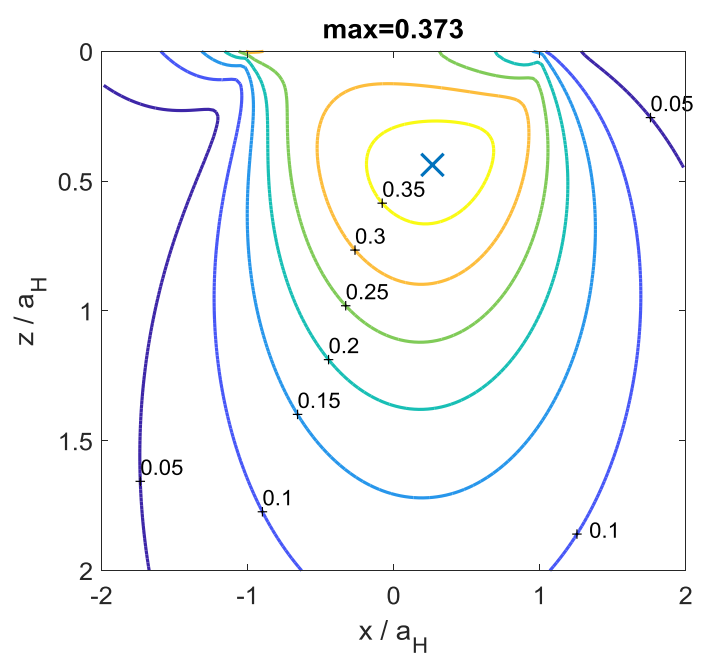

(b)

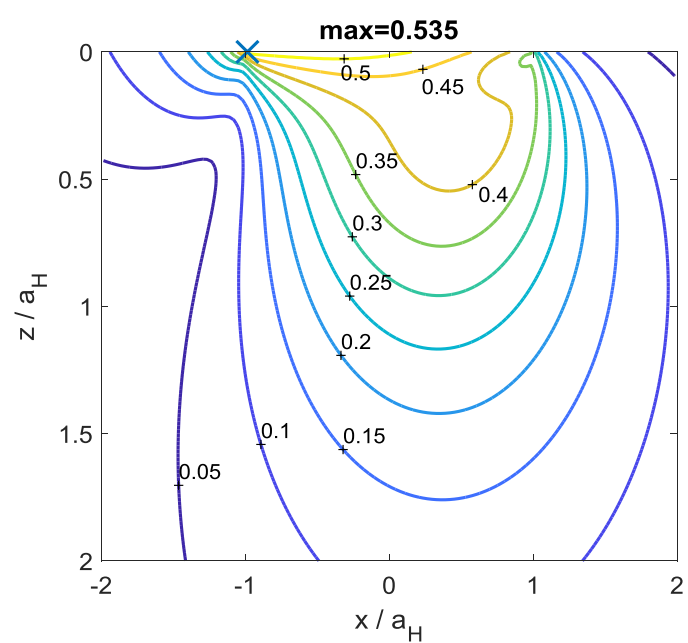

(c)

Fig. 4. Iso-contours of $\sqrt{J_{2}} / p_{H}$ in the plane $y=0$, $E_{1}=E_{2}$ : (a) $\mu=0$ (Hertz); (b) $\mu=0.25$; (c) $\mu=0.5$. 


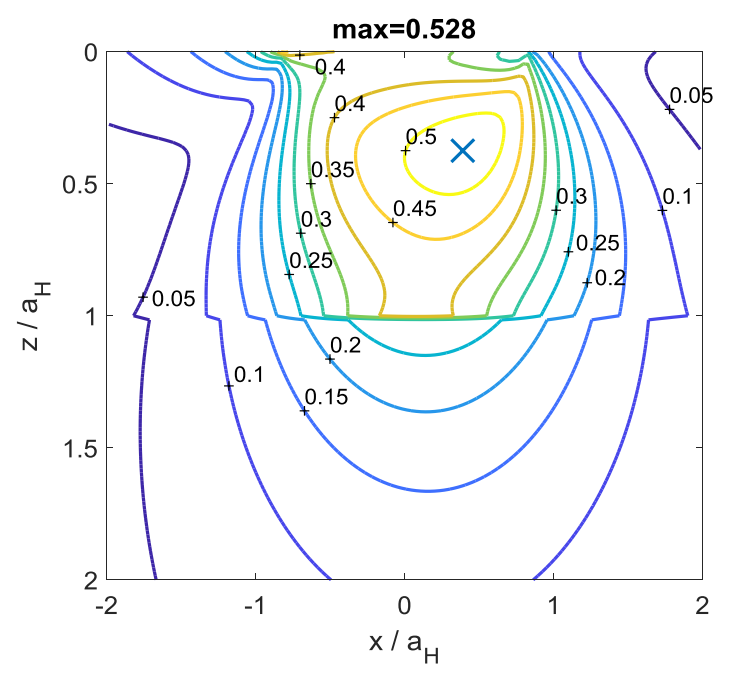

(a)

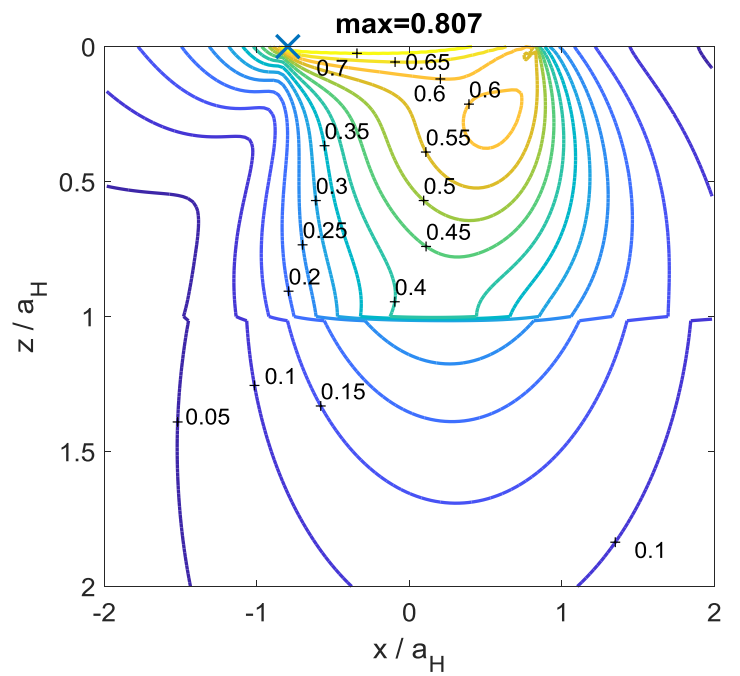

(b)

Fig. 5. Iso-contours of $\sqrt{J_{2}} / p_{H}$ in the plane $y=0$, $E_{1} / E_{2}=2, h / a_{H}=1:$ (a) $\mu=0.25$, (b) $\mu=0.5$.

A parametric study, aiming to assess the combined influence of the coating thickness $h$, of the frictional coefficient $\mu$ and of the elastic modulus mismatch $E_{1} / E_{2}$, on the intensity and position of the maximum equivalent stress, is performed next. To this end, the ratio $E_{1} / E_{2}$ is varied between 0.5 and 4 with a step of 0.5 , $h / a_{H}$ between 0.25 and 1.5 with a step of 0.25 , and $\mu$ between 0.1 and 0.6 . For the latter parameter, a 0.05 increment is imposed, except for the interval $[0.3,0.4]$, in which a smaller increment of 0.02 is considered. This mesh refinement is intended to capture more accurately the snap of the maximum stress from depth to the surface. A set of $8 \times 6 \times 14$ contact simulations is thus performed, and the results are depicted in Figs. 7-9.

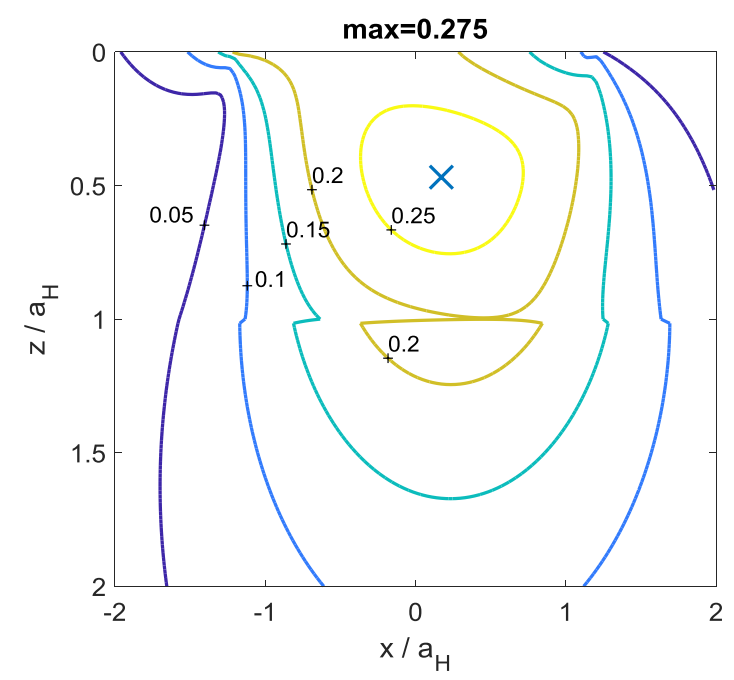

(a)

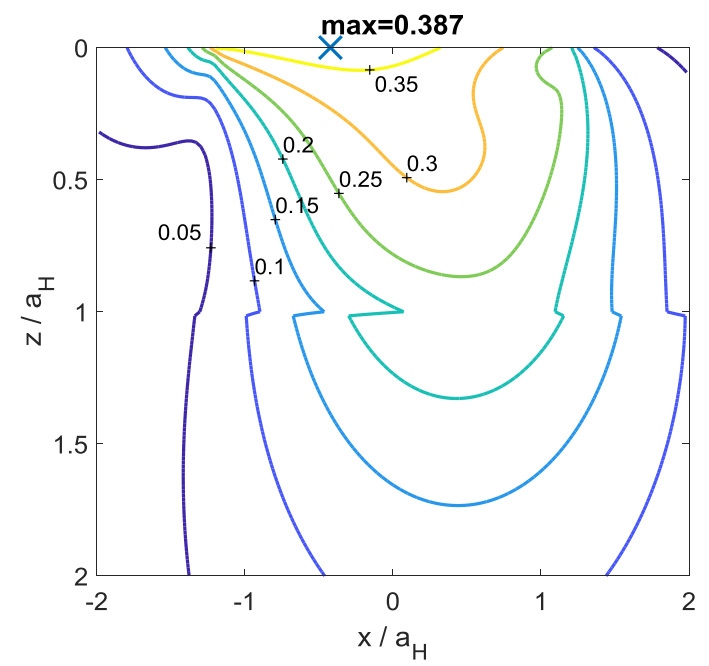

(b)

Fig. 6. Iso-contours of $\sqrt{J_{2}} / p_{H}$ in the plane $y=0$, $E_{1} / E_{2}=1 / 2, h / a_{H}=1:$ (a) $\mu=0.25$; (b) $\mu=0.5$.

Figure 7 illustrates the contour plots of the intensity of the maximum equivalent stress $\sqrt{J_{2}} / p_{H}$. Calculation of the latter parameter is essential to study the plastic flow or the crack nucleation in the coated contact. The critical load condition for a plasticity contact failure is usually evaluated by equating the von Misses stress with the yield strength. Mapped data of the maximum equivalent stress intensity may lead to a rapid assessment of the potential yield locations. The contour plots suggest that, in all cases, the normalized maximum equivalent stress increases with both the frictional coefficient and the elastic modulus mismatch $E_{1} / E_{2}$, reaching intensities of $1.5 p_{H}$, almost five times higher than the value for the Hertz frictionless and uncoated contact. Thicker coatings lead to higher stress intensities. 


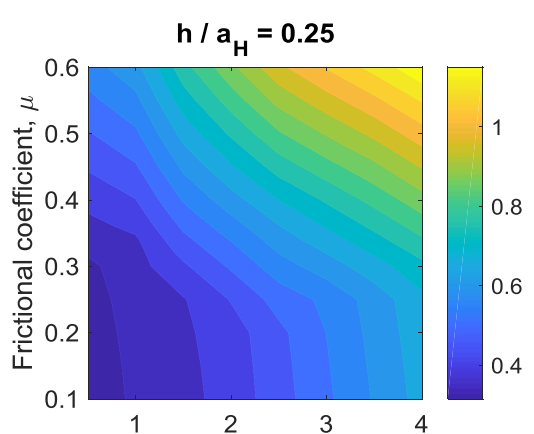

Elastic modulus mismatch, $E_{1} / E_{2}$

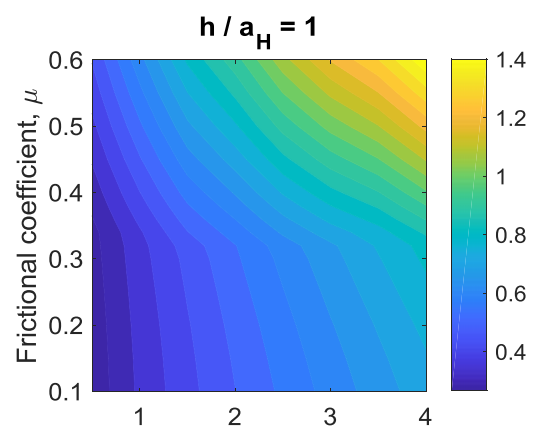

Elastic modulus mismatch, $E_{1} / E_{2}$

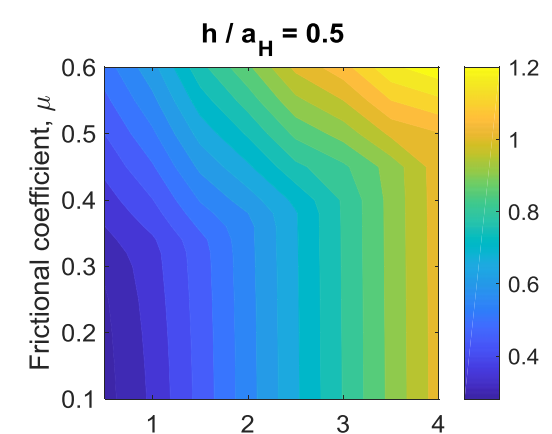

Elastic modulus mismatch, $\mathrm{E}_{1} / \mathrm{E}_{2}$

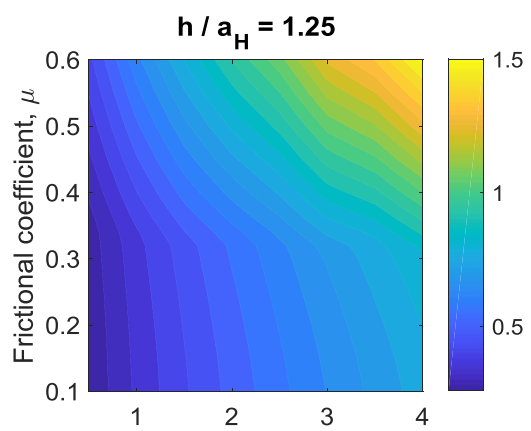

Elastic modulus mismatch, $\mathrm{E}_{1} / \mathrm{E}_{2}$

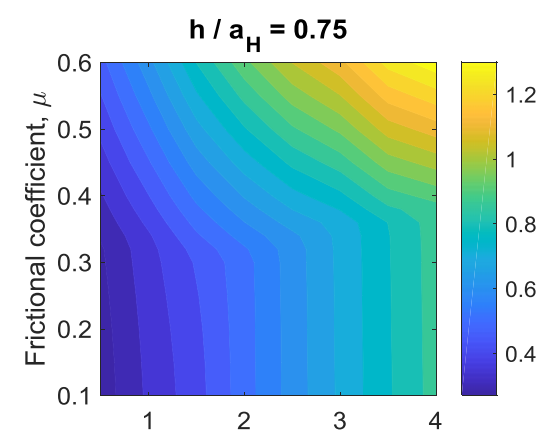

Elastic modulus mismatch, $E_{1} / E_{2}$

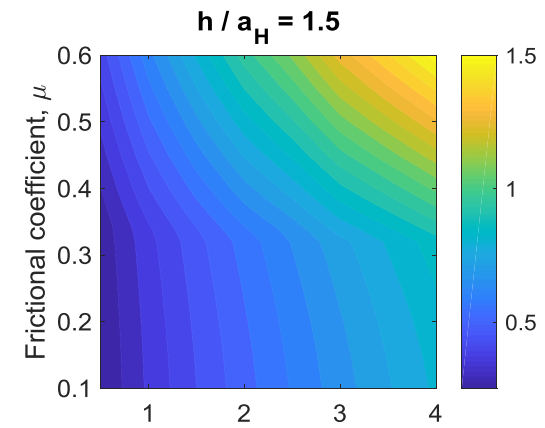

Elastic modulus mismatch, $\mathrm{E}_{1} / \mathrm{E}_{2}$

Fig. 7. Iso-contours of the intensity of the maximum $\sqrt{J_{2}} / p_{H}$.

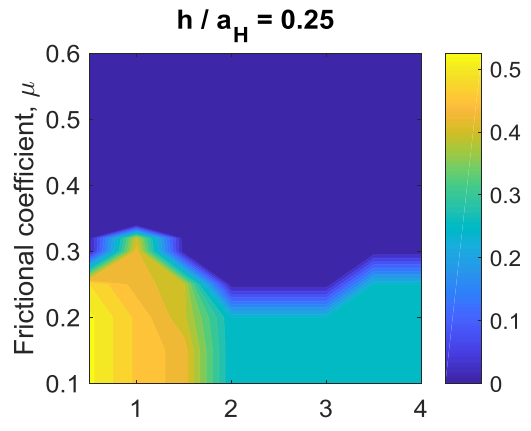

Elastic modulus mismatch, $\mathrm{E}_{1} / \mathrm{E}_{2}$

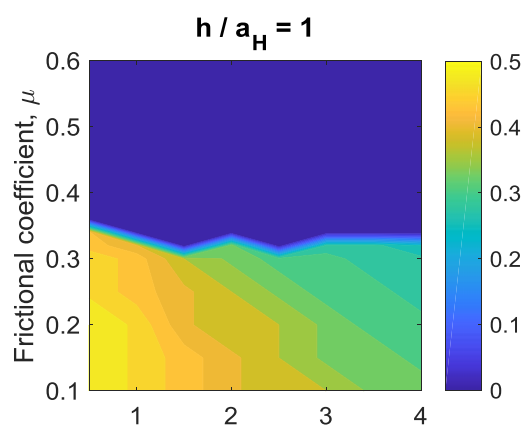

Elastic modulus mismatch, $\mathrm{E}_{1} / \mathrm{E}_{2}$

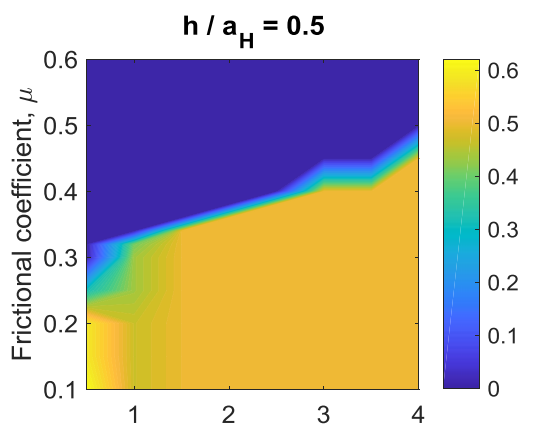

Elastic modulus mismatch, $\mathrm{E}_{1} / \mathrm{E}_{2}$

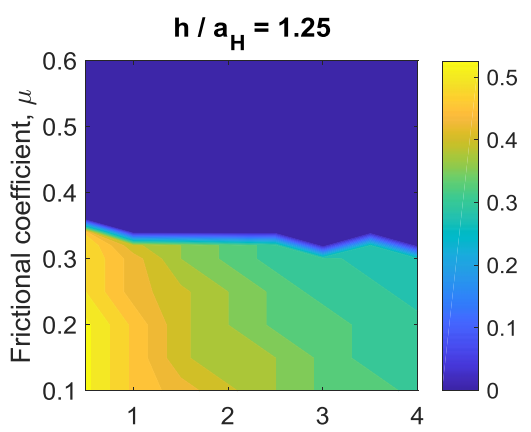

Elastic modulus mismatch, $E_{1} / E_{2}$

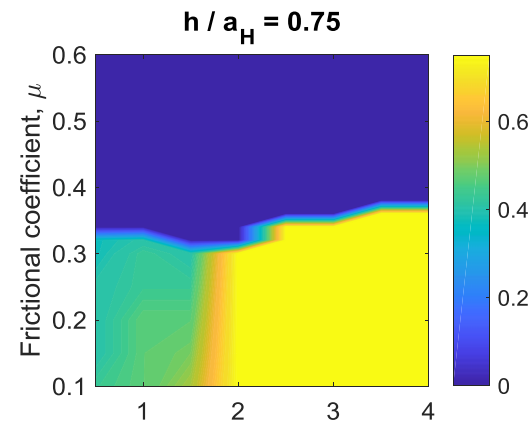

Elastic modulus mismatch, $\mathrm{E}_{1} / \mathrm{E}_{2}$

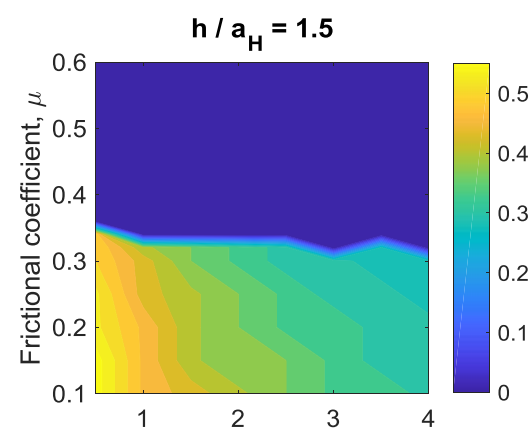

Elastic modulus mismatch, $\mathrm{E}_{1} / \mathrm{E}_{2}$

Fig. 8. Iso-contours of the dimensionless depth of the maximum $\sqrt{J_{2}} / p_{H}$. 


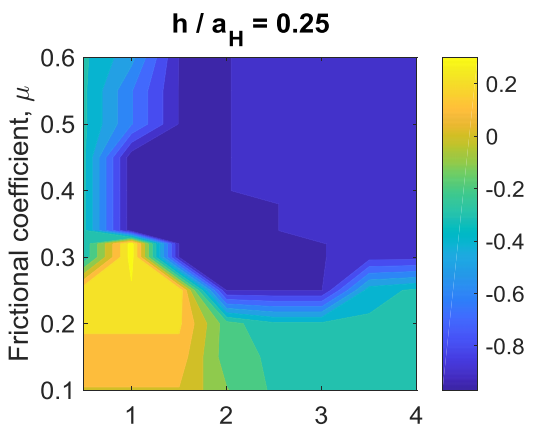

Elastic modulus mismatch, $\mathrm{E}_{1} / \mathrm{E}_{2}$

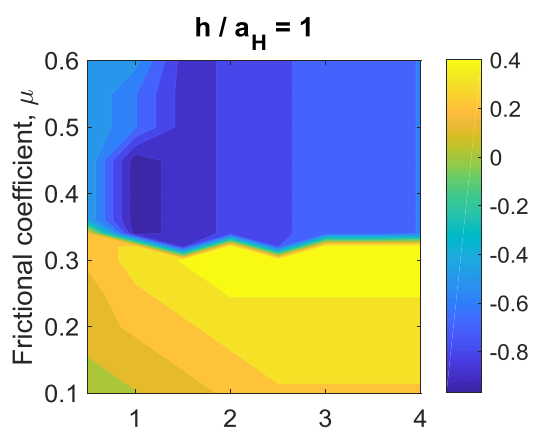

Elastic modulus mismatch, $\mathrm{E}_{1} / \mathrm{E}_{2}$

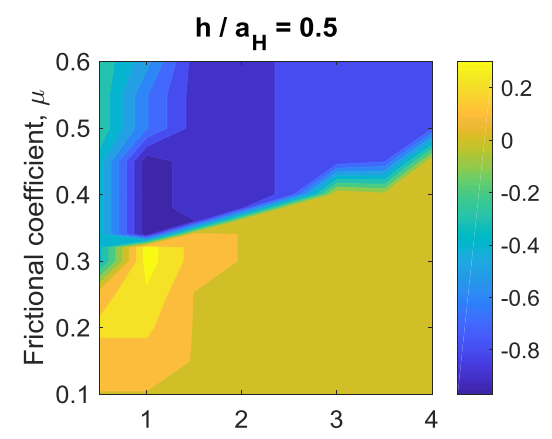

Elastic modulus mismatch, $\mathrm{E}_{1} / \mathrm{E}_{2}$

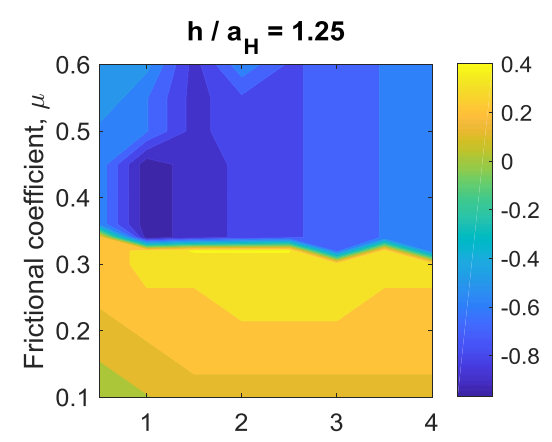

Elastic modulus mismatch, $\mathrm{E}_{1} / \mathrm{E}_{2}$

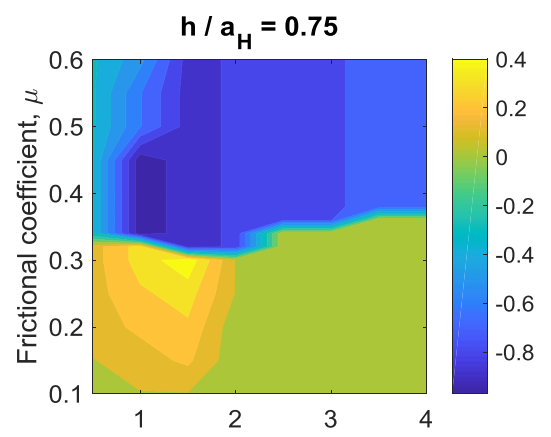

Elastic modulus mismatch, $\mathrm{E}_{1} / \mathrm{E}_{2}$

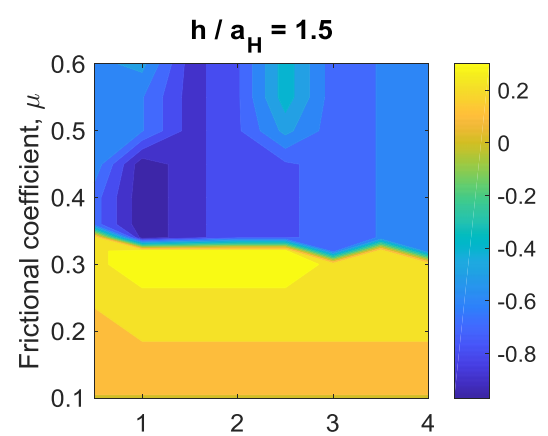

Elastic modulus mismatch, $\mathrm{E}_{1} / \mathrm{E}_{2}$

Fig. 9. Iso-contours of the dimensionless $\mathrm{x}$-coordinate of the maximum $\sqrt{J_{2}} / p_{H}$.

The setting of the maximum stress is also important for design decisions. The literature [17-19] has proven that cracks are probable to originate in thinner coatings due to the location of maximum shear stress close to the interface between the coating and the substrate. The depth of the maximum stress, normalized by $a_{H}$, is depicted in Fig. 8. The plots suggest that, except for the very thin coating, a frictional coefficient greater than 0.3 moves the maximum stress from depth to the boundary. The greatest depth is predicted for the lowest frictional coefficient and the lowest ratio $E_{1} / E_{2}$, except for the case $h / a_{H}=0.75$, when the maximum is located close to the interface for $E_{1} / E_{2}>2$ and $\mu<0.3$.

Figure 9 depicts the contour plots of the $x$ coordinate of the maximum stress, normalized by $a_{H}$. For lower frictional regimes, the maximum is moved toward the leading edge of the contact (i.e., $x>0$ ), whereas higher friction results in maximums located toward the trailing edge of the contact (i.e., $x<0$ ). Considering the superposition of the iso-contours in Figs. 8 and 9 , it can be concluded that subsurface maximums have positive $x$ coordinates, whereas surface maximums possess negative radial extents, reaching the contact radius in the direction opposing the sliding motion.

\section{CONCLUSIONS}

A state-of-the-art contact solver assisted by the conjugate gradient method is combined with a numerical technique for calculation of displacement in bilayer materials based on existing analytical responses to unit point loadings available in the Fourier transform domain, yielding a fast and accurate numerical tool for the analyses of stresses arising in coated contacts.

Using the novel computer program, a parametric study on the combined influence of the coating thickness, frictional coefficients and elastic modulus mismatch, on the maximum intensity of the von Mises equivalent stress and its location, is performed for a wide range of input parameters, with increments small enough as to accurately capture rapid changes in the output. A novel map of the possible yield locations in coated contacts is thus advanced, which can be used for quick estimation of the optimum coating design without contact failure. 
The parametric study suggests that the stress intensity increases with the frictional coefficient, with the elastic modulus mismatch and with the coating thickness. Lower frictional regimes position the maximum in depth, toward the leading contact boundary, whereas an increase of the frictional coefficient beyond 0.3 shifts the maximum to the free surface, toward the trailing contact boundary.

The proposed numerical method presents itself as a robust tool for contact analysis, capable of advancing the understanding and prediction of coated contact processes for which analytical solutions are lacking and other numerical techniques are computationally intensive.

\section{REFERENCES}

[1] Y. Ju, T.N. Farris, Spectral Analysis of TwoDimensional Contact Problems, Journal of Tribology, vol. 118, iss. 2, pp. 320-328, 1996, doi: $10.1115 / 1.2831303$

[2] T.C. O'Süllivian, R.B. King, Sliding Contact StressField due to a Spherical Indenter on a Layered Elastic Half-space, Journal of Tribology, vol. 110, iss. 2, pp. 235-240, 1988, doi: 10.1115/1.3261591

[3] T. Nogi, T. Kato, Influence of a Hard Surface Layer on the Limit of Elastic Contact-Part I: Analysis Using a Real Surface Model, Journal of Tribology, vol. 119, iss. 3, pp. 493-500, 1997, doi: $10.1115 / 1.2833525$

[4] I.A. Polonsky, L.M. Keer, A Fast and Accurate Method for Numerical Analysis of Elastic Layered Contacts, Journal of Tribology, vol. 122, iss. 1, pp. 30-35, 1999, doi: 10.1115/1.555323

[5] I.A. Polonsky, L.M. Keer, Stress Analysis of Layered Elastic Solids With Cracks Using the Fast Fourier Transform and Conjugate Gradient Techniques, Journal of Applied Mechanics, vol. 68, iss. 5, pp. 708-714, 2001, doi: $10.1115 / 1.1381394$

[6] S.B. Liu, Q. Wang, G. Liu, A Versatile Method of Discrete Convolution and FFT (DC-FFT) for Contact Analyses, Wear, vol. 243, iss. 1-2, pp. 101-111, 2000, doi: 10.1016/S0043-1648(00)00427-0

[7] S. Liu, Q. Wang, Studying Contact Stress Fields Caused by Surface Tractions With a Discrete Convolution and Fast Fourier Transform Algorithm, Journal of Tribology, vol. 124, iss. 1, pp. 36-45, 2001, doi: 10.1115/1.1401017

[8] Z.J. Wang, W.-Z. Wang, H. Wang, D. Zhu, Y.-Z. Hu, Partial Slip Contact Analysis on Three-
Dimensional Elastic Layered Half Space, Journal of Tribology, vol. 132, iss. 2, pp. 021403-1-12, 2010, doi: 10.1115/1.4001011

[9] P.Y. Zhang, D.F. Diao, Z.J. Wang, ThreeDimensional Local Yield Maps of Hard Coating Under Sliding Contact, Journal of Tribology, vol. 134, iss. 2, pp. 021301-1-8, 2012, doi: $10.1115 / 1.4005265$

[10] C. Yu, Z. Wang, G. Liu, L.M. Keer, Q.J. Wang, Maximum von Mises Stress and Its Location in Trilayer Materials in Contact, Journal of Tribology, vol. 138, iss. 4, pp. 041402-1-13, 2016, doi: 10.1115/1.4032888

[11] C. Yu, Z. Wang, Q.J. Wang, Analytical Frequency Response Functions for Contact of Multilayered Materials, Mechanics of Materials, vol. 76, pp. 102-120, 2014, doi: 10.1016/j.mechmat.2014.06.006

[12] I.A.Polonsky, L.M. Keer, A Numerical Method for Solving Rough Contact Problems Based on the Multi-Level Multi-Summation and Conjugate Gradient Techniques, Wear, vol. 231, iss. 2, pp. 206-219, 1999, doi: 10.1016/S00431648(99)00113-1

[13] J.J. Kalker, Y. Van Randen, A Minimum Principle for Frictionless Elastic Contact with Application to Non-Hertzian Half-Space Contact Problems, Journal of Engineering Mathematics, vol. 6, iss. 2, pp. 193-206, 1972, doi: 10.1007/BF01535102

[14] K.L. Johnson, Contact Mechanics, Cambridge University Press, Cambridge, 1985.

[15] J. Allwood, Survey and Performance Assessment of Solution Methods for Elastic Rough Contact Problems, Journal of Tribology, vol. 127, iss. 1, pp. 10-23, 2005, doi: 10.1115/1.1828073

[16] D.M. Burmister, The General Theory of Stresses and Displacements in Layered Systems. I, Journal of Applied Physics, vol. 16, pp. 89-94, 1945, doi: 10.1063/1.1707558

[17] L.M. Keer, R.E. Worden, A Qualitative Model to Describe the Microchipping Wear Model in Ceramic Rollers, Tribology Transactions, vol. 33, iss. 3, pp. 411-417, 1990, doi: 10.1080/10402009008981971

[18] L.M. Keer, C.H. Kuo, Cracking in a Loaded Brittle Elastic Half-Space, International Journal of Solids and Structures, vol. 29, iss. 14-15, pp. 1819-1826, 1992, doi: 10.1016/00207683(92)90173-Q

[19] A.F Bower, N.A Fleck, Brittle Fracture Under a Sliding Line Contact, Journal of the Mechanics and Physics of Solids, vol. 42, iss. 9, pp. 13751396, 1994, doi: 10.1016/00225096(94)90002-7 


\section{APPENDIX}

The constituents of the FRFs needed for the computation of the shear-induced stresses, as derived in [7], are given below:

$$
\begin{gathered}
\alpha=\sqrt{\zeta^{2}+\xi^{2}} \\
\varepsilon^{(i)}=e^{\alpha z_{i}} ; \bar{\varepsilon}^{(i)}=e^{-\alpha z_{i}} ; \mu=G_{1} / G_{2} ; \\
v=\left(1-v_{2}\right) /\left(1-v_{1}\right) ; \\
\kappa=(\mu-1) /\left(\mu+3-4 v_{1}\right) ; \\
\beta_{1}=-1 /\left\{2 \alpha\left(1-v_{1}\right)\left[(1+\mu)+(1-\mu) e^{-2 \alpha h}\right]\right\} ; \\
\beta_{2}=2 \sqrt{-1} \zeta \beta_{1} /(v \alpha) ; \\
B^{(1)}=\beta_{1}(\mu+1) ; \\
\bar{B}^{(1)}=\beta_{1}(\mu-1) e^{-2 \alpha h} ; \\
B^{(2)}=2 \beta_{1} e^{-\alpha h} / v ; \\
\lambda_{0}=1-4\left(1-v_{1}\right) /\left[1+\mu\left(3-4 v_{2}\right)\right] ; \\
\lambda_{1}=-\sqrt{-1} \zeta \alpha^{-1}\left(B^{(1)}-\bar{B}^{(1)}\right) ; \\
\lambda_{2}=-2 \sqrt{-1} \zeta\left(1-v_{1}\right)\left(B^{(1)}+\bar{B}^{(1)}\right) / \alpha ; \\
\lambda_{3}=2 \sqrt{-1} \zeta \beta_{1}[(1-v) /(v \alpha)+h \mu] ; \\
\lambda_{4}=2 \sqrt{-1} \zeta \beta_{1}\left[2\left(1-v_{2}\right)(1-\mu) /(v \alpha)+h\right]
\end{gathered}
$$

$$
\begin{gathered}
\lambda_{5}=2 \sqrt{-1} \zeta \beta_{1} h ; \\
\lambda_{6}=2 \sqrt{-1} \zeta \beta_{1}[(\mu-v) /(v \alpha)+h \mu] ; \\
\gamma_{1}=\left(2 v_{2}-1\right) \beta_{2} /\left[1+\mu\left(3-4 v_{2}\right)\right] ; \\
\gamma_{2}=\left[\left(\lambda_{2}-\lambda_{1}\right)\left(\lambda_{0}+2 \alpha h\right)+\lambda_{1}+\lambda_{2}-2 \lambda_{4}+\right. \\
\left.4\left(1-v_{2}\right)\left(\mu \gamma_{1}+\beta_{2}\right)\right] e^{-2 \alpha h} \times \\
{\left[2\left(\lambda_{0} e^{-2 \alpha h}-1+2 \alpha h e^{-2 \alpha h}\right)\right]^{-1}} \\
\gamma_{3}=\left[\lambda_{4}+\lambda_{3}-\lambda_{2}-\lambda_{1}-\left(3-2 v_{2}\right) \beta_{2}+2 \alpha h \times\right. \\
\left.\left(2 \gamma_{2}-\lambda_{2}+\lambda_{1}\right)\right] \times \\
{\left[1+\lambda_{0} \kappa e^{-4 \alpha h}-\left(\lambda_{0}+\kappa+4 \alpha^{2} h^{2} \kappa\right) e^{-2 \alpha h}\right]^{-1} ;} \\
C^{(1)}=\gamma_{3} e^{-2 \alpha h}\left(1-\kappa e^{-2 \alpha h}-2 \alpha h \kappa\right)-2 \gamma_{2}+\lambda_{2}-\lambda_{1} ; \\
\bar{C}^{(1)}=\gamma_{3} e^{-2 \alpha h} \kappa\left(\lambda_{0} e^{-2 \alpha h}+2 \alpha h e^{-2 \alpha h}-1\right) ; \\
C^{(2)}=e^{-\alpha h}\left[C^{(1)}\left(1-\lambda_{0}\right)+(1-\mu) \gamma_{1}\right] ; \\
D^{(1)}=\left[\lambda_{1}+\lambda_{2}-\left(3-4 v_{1}\right) C^{(1)}+\bar{C}^{(1)}\right] /(2 \alpha) ; \\
\bar{D}^{(1)}=\left[\lambda_{2}-\lambda_{1}-C^{(1)}+\left(3-4 v_{1}\right) \bar{C}^{(1)}\right] /(2 \alpha) ; \\
D^{(2)}=-\bar{C}^{-\alpha h}\left[C^{(1)}\left(3-4 v_{2}\right)\left(1-\lambda_{0}\right)+\gamma_{3} \times\right. \\
\left(\lambda_{0} e^{-2 \alpha h}+2 \alpha h e^{-2 \alpha h}-1\right)(\kappa-1)+4\left(1-v_{2}\right) \times \\
\left.\left(\beta_{2}+\gamma_{1}\right)\right](2 \alpha)^{-1} ;
\end{gathered}
$$

